\title{
ELRIS2D: A MATLAB Package for the 2D Inversion of DC Resistivity/IP Data
}

\author{
Irfan AKCA \\ Ankara University, Faculty of Engineering, \\ Department of Geophysical Engineering, Ankara, Turkey; \\ e-mail: iakca@eng.ankara.edu.tr
}

\begin{abstract}
ELRIS2D is an open source code written in MATLAB for the twodimensional inversion of direct current resistivity (DCR) and time domain induced polarization (IP) data. The user interface of the program is designed for functionality and ease of use. All available settings of the program can be reached from the main window. The subsurface is discretized using a hybrid mesh generated by the combination of structured and unstructured meshes, which reduces the computational cost of the whole inversion procedure. The inversion routine is based on the smoothness constrained least squares method. In order to verify the program, responses of two test models and field data sets were inverted. The models inverted from the synthetic data sets are consistent with the original test models in both DC resistivity and IP cases. A field data set acquired in an archaeological site is also used for the verification of outcomes of the program in comparison with the excavation results.
\end{abstract}

Key words: 2D inversion, GUI, finite elements, hybrid mesh.

\section{INTRODUCTION}

Direct current resistivity (DCR) method is one of the most frequently used geophysical tools for the solution of near surface problems. Impressive developments occur in the multichannel resistivity measurement technologies,

Ownership: Institute of Geophysics, Polish Academy of Sciences;

(C) 2016 Akca. This is an open access article distributed under the Creative Commons

Attribution-NonCommercial-NoDerivs license,

http://creativecommons.org/licenses/by-nc-nd/3.0/. 
thus enabling thousands of readings in a few hours. This ability leads to explore a survey site in more details, increasing the volume of the data to be processed. An exhaustive review about the recent developments in DCR imaging may be found in the paper by Loke et al. (2013) where some applications for a variety of survey objectives are included. Although threedimensional (3D) data inversion and interpretation techniques are available (e.g., Pidlisecky et al. 2007), two-dimensional interpretation of DCR and IP data is still a valuable option. Two-dimensional inversion of DCR data is an extensively-studied research area which produced several commercial and academic inversion codes and software (i.e., Res2DInv 2014, EarthImager 2009, DC2DInvRes 2014). These packages have their own originalities with plenty of customization options and routines for the inversion and visualization of DCR/IP data. In addition to the above-mentioned packages, there are several open source codes (i.e., Karaoulis et al. 2013, Pidlisecky and Knight 2008) developed for different purposes.

This paper presents a new open source two-dimensional inversion program named ELRIS2D. The novelty of the program arose from the discretization routine involving the hybrid use of structured and unstructured meshes and the design of the user interface. ELRIS2D provides a simple and convenient user interface designed for the ease of use to invert and visualize $\mathrm{DCR} / \mathrm{IP}$ data which is written in MATLAB. All available options can be reached from the main window by clicking on the corresponding user interface element. This approach in the design of user interface speeds up the process of multiple data sets. A hybrid mesh application for the finiteelement method reduces the computational requirements and consumed CPU time. ELRIS2D uses the smoothness constrained least squares method (Loke and Barker 1996, Tsourlos et al. 1998) for the definition of objective function to be minimized. The parameter correction vector is calculated by solving the linear system of equations via the MATLAB built-in function "mldivide". A variety of techniques have been suggested for the incorporation of a smoothing operator to stabilize the inversion process and extract structural information from the geolectrical models (see Zhou et al. 2014, Akca and Başokur 2010, Başokur and Akca 2011). A five point Laplacian operator is used as the smoothness constraint in ELRIS2D for simplicity. The user interface provides some options and tools to enhance the view of the data and model sections. Outputs of the program may be exported as a snapshot, pdf or text files. ELRIS2D was tested with the responses of synthetic models and a field data set measured at the ancient city of Pisidian Antioch, in western Turkey. Test results show that the new open source code ELRIS2D is a powerful tool for the detection of subsurface targets and additional functionalities can easily be added by other researchers. 


\section{DESIGN AND IMPLEMANTATION}

\subsection{Mesh generation}

Two-dimensional finite element meshes are usually constructed using triangular elements that provide more flexibility for the representation of known interfaces among subsurface units. Most commonly used finite element meshes are classified as "structured" and "unstructured" according to the placement and shape of the elements. Figure 1a,b illustrates structured and unstructured triangular mesh examples used in 2D modeling and inversion of DCR data. Structured mesh generation is based on dividing the rectangular (or tetragonal) elements into two or four triangles (Fig. 1b), whilst unstructured meshes are generated following the Delaunay triangulation rules constrained by the triangle quality (Fig. 1a). Shewchuk (1997) and Si (2008) gave comprehensive description of unstructured mesh design in two and three dimensions, and Rücker (2011) reviews the usage of unstructured meshes in DCR modeling and inversion. Among many others, the main advantage of using an unstructured mesh is reducing the size of the mesh and thus governing matrices used in forward calculations. Additionally, it is a flexible tool to outline irregular interfaces precisely while it permits the use of greater sized triangles in parts of the model mesh where the data sensitivity is low. The unstructured mesh given in Fig. 1a consists of 363 nodes which is nearly half the number of nodes of the structured mesh designed for the discretization of the same model. The number of triangles forming the structured mesh is nearly three times greater than that of the unstructured mesh.

Besides the abovementioned advantages of the unstructured meshes, some problems arise when they are used to parameterize an unknown subsurface model. For example, the arrangement of the triangles is somewhat irregular, which may cause flower or star shaped bodies to appear in the inverted model sections. This case gives rise to difficulties in the interpretation of the resistivity sections. For a better understanding of model and finite element mesh construction, it will be helpful to remember the following basic aspects regarding the discretization of subsurface.

Discretization of the subsurface is essential to the fulfillment of two requirements:

(1) A conceptual geophysical model is required to present the subsurface distribution of a physical parameter with certain geometry;

(2) Finite elements solution requires a bounded and a fine discretized domain.

The fulfillment of the two requirements listed above with totally unstructured or structured meshes causes the unnecessary growth of the mesh size (Fig. 1a,b). Therefore, some researchers distinguish parameter and calculation meshes. For example, Günther (2004) and Rücker(2011) suggest a triple 


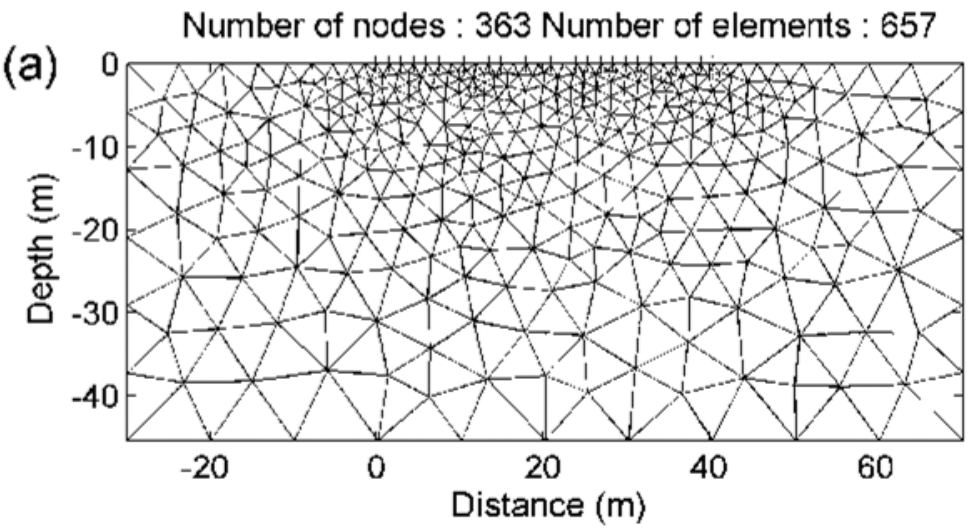

Number of nodes : 648 Number of elements : 1785
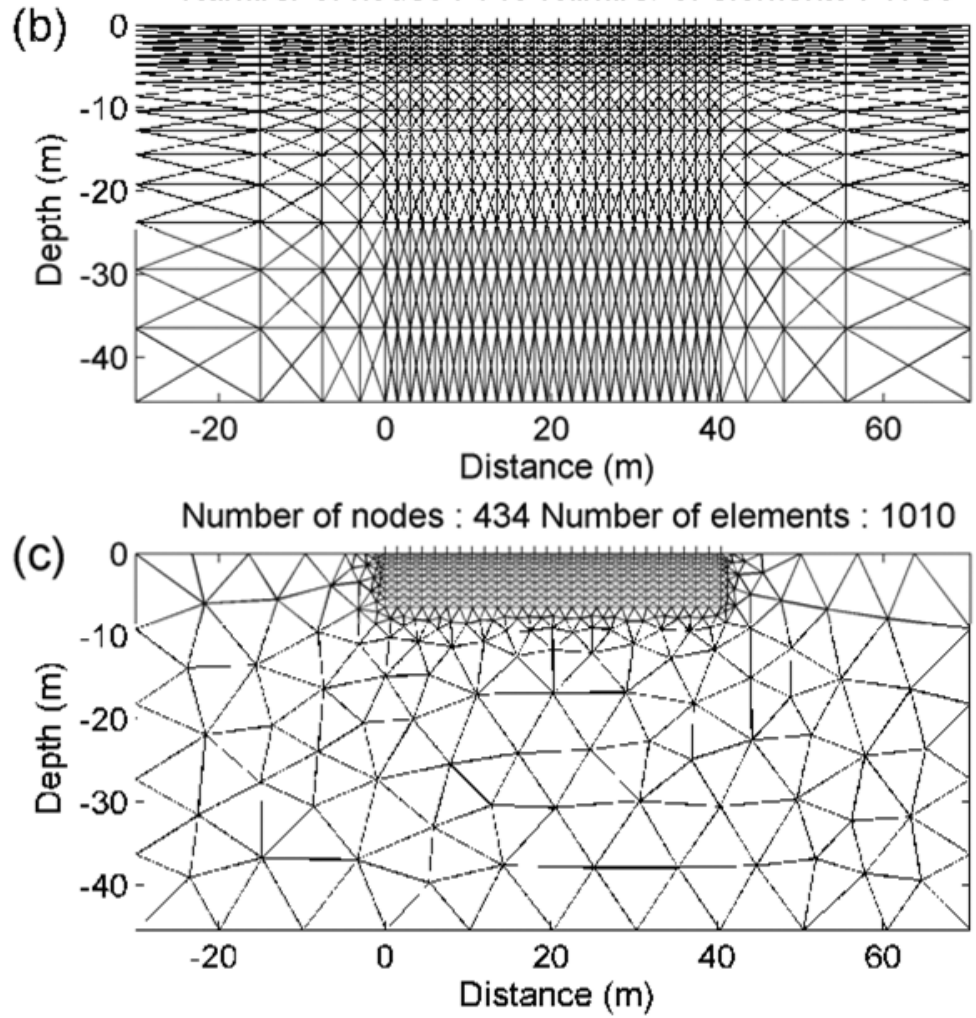

Fig. 1: (a) Unstructured, (b) structured, and (c) hybrid finite element meshes.

grid technique where three different unstructured meshes were used to "combine a resolution-dependent model parameterization with accurate forward calculations using finite elements". 
In the present paper, a combination of regular and unstructured finite element meshes is used to employ the advantages of both types of meshes. The model mesh is divided into two subdomains. The first subdomain bounds the model region where measured data are sufficiently sensitive to solve the model parameters with high fidelity. The model region is bounded by the first and last electrode positions in $x$-direction and with maximum depth of investigation in $z$-direction. The maximum depth of penetration is calculated according to Edwards (1977). This portion of the mesh is constructed by dividing regularly arranged rectangles into triangular elements and each rectangular cell is treated as a model parameter. The remaining part of the mesh is required for the numerical implementation of finite elements algorithm and designed by using triangular elements with enlarging size towards the outer boundaries of the mesh (Fig. 1c). This leads to the reduction of the mesh size compared to a structured mesh keeping the conventional arrangement of the rectangular model cells. An example of the mentioned approach may also be seen in the tutorial by Günther and Rücker (2015). The unstructured portion of the mesh is produced by the program developed by Shewchuk (1997).

\subsection{Forward modeling}

The following elliptic equation is solved to calculate the potential distribution $\phi(x, y, z)$ over a $2 \mathrm{D}$ conductivity structure $(\sigma)$ due to a current source $(I)$ :

$$
-\nabla[\sigma(x, z) \nabla \phi(x, y, z)]=I(x, y, z) .
$$

Equation 1 governs the three-dimensional potential distribution over a model where the conductivities differ only in $x$ - and $z$-directions. The numerical solution of Eq. 1 may be achieved by the use of a numerical approach such as finite difference (Mufti 1976, Dey and Morrison 1979) or finite elements (Coggon 1971, Rijo 1977, Pelton et al. 1978). Solution of Eq. 1 is discussed by a plenty of researchers and well understood. Therefore, the paper will focus on the subsurface discretization and practical aspects of the application of finite elements method used in the program.

Application of finite element method to Eq. 1 applying Neumann and Dirichlet boundary conditions at the boundaries of a discretized domain results in a linear system of equations expressed as:

$$
\mathbf{K} \cdot \mathbf{v}=\mathbf{S},
$$

where $\mathbf{K}$ is a positive definite, symmetric band matrix, $\mathbf{v}$ is a column vector comprising the unknown potentials at each mesh node, and $\mathbf{S}$ is the source term. Equation 2 can be solved by a matrix division in MATLAB as: $v=K \backslash S$ using Gaussian elimination. The matrix division operator in MATLAB al- 
lows the solution of potentials due to all possible single current sources at once. Therefore, each column of $\mathbf{S}$ includes one nonzero element corresponding to the strength of the virtual current source which is set to $2 \pi A$. Other elements of the source matrix are set as 0 . Although the numerical calculations are based on conductivity and potential distribution, in practice the potential difference and apparent resistivity quantities are used. Therefore, the potential differences should be converted to apparent resistivities based on the layout of electrodes. Marescot et al. (2006) gave a comprehensive description of approaches for the definition of apparent resistivities. The definition called "general approach" is given by:

$$
\rho_{a}=\frac{\Delta V}{\Delta V_{0}} \rho_{0},
$$

where $\Delta V_{0}$ and $\Delta V$ are respective potential differences for a homogeneous half-space with resistivity $\rho_{0}$ and a model with arbitrary resistivity distribution. $\rho_{0}$ may be assigned as $1 \mathrm{ohm}-\mathrm{m}$ for simplicity. This yields in a normalized potential difference with the response of system to the unity. The potentials at each node are calculated by assuming each electrode acts as a current pole. Therefore, any combination of current-potential electrodes may be used to calculate the apparent resistivity values. The apparent resistivity values are demonstrated as pseudosections following the basis given by Edwards (1977).

In the IP forward modeling an additional parameter, namely chargeability $(\eta)$, is introduced to describe the polarization of subsurface (Seigel 1959). Chargeability is a physical parameter that can be used to explain the microscopic IP phenomena in a macroscopic manner (Oldenburg and Li 1994). I refer to Seigel (1959), Sumner (1976), Bertin and Loeb (1976), Pelton et al. (1978), Fink et al. (1990), and Ward (1990) for the basics, case histories, and mathematical background of forward modeling and inversion of IP data.

The response of a 2D chargeability model $\eta(x, z)$ can be calculated by calling the DC resistivity forward operator $F_{\mathrm{dc}}$ twice for the conductivity models $\sigma(1-\eta)$ and $\sigma$, respectively, and evaluating the following equation:

$$
\eta_{a}=\frac{F_{\mathrm{dc}}[\sigma(1-\eta)]-F_{\mathrm{dc}}[\sigma]}{F_{\mathrm{dc}}[\sigma(1-\eta)]},
$$

where $\eta_{a}$ is the apparent chargeability.

\subsection{Inversion}

Inversion of DC resistivity data is a non-linear and ill-posed problem. An initial model supplied by the user is updated by an iterative process. Usually, a kind of model smoothness constraint is required to stabilize the inversion. 
The model smoothness constraints link the neighboring model parameters (resistivity of adjacent rectangular cells) in order to prevent sharp changes in the individual resistivities which may result in a meaningless geoelectrical section. The inversion routine used in the program is based on the solution of the following equation (Loke 2014, Wolke and Schwetlick 1988, Farquharson and Oldenburg 1998):

$$
\Delta \mathbf{m}_{\mathbf{i}}=\left(\mathbf{J}^{\mathrm{T}} \mathbf{W}_{\mathrm{d}}^{\mathrm{T}} \mathbf{W}_{\mathrm{d}} \mathbf{J}+\lambda \mathbf{C}\right)^{-1}\left(\mathbf{J}^{\mathrm{T}} \mathbf{W}_{\mathrm{d}}^{\mathrm{T}} \mathbf{W}_{\mathrm{d}} \Delta \mathbf{d}-\lambda \mathbf{C} \mathbf{m}_{i-1}\right),
$$

where $\Delta \mathbf{m}_{\mathbf{i}}$ is the model correction vector, $\mathbf{J}$ is the Jacobian matrix, $\lambda$ is the regularization parameter, $\Delta \mathbf{d}$ is the data discrepancy vector, $i$ denotes the iteration number, $\mathbf{W}_{\mathrm{d}}$ is data weighting matrix, and $\mathbf{C}$ is the five-point finite difference Laplacian. The data weighting matrix is in the given form:

$$
\mathbf{W}_{\mathrm{d}}=\operatorname{diag}\left(1 / \boldsymbol{\varepsilon}_{\mathbf{i}}\right),
$$

where $\boldsymbol{\varepsilon}_{\mathbf{i}}$ denotes the individual standard deviation of the measurements in case of availability. Otherwise, the data weighting matrix is calculated by the following equation:

$$
\mathbf{W}_{\mathrm{d}}=\operatorname{diag}\left(\frac{1}{\sqrt[4]{\boldsymbol{\rho}_{\text {app }}}}\right) .
$$

The data weighting procedure aims to reduce the effects of extreme readings to the inversion. The smoothness operator $\mathrm{C}$ is formed by using the "delsq" MATLAB function. The function requires key numbers of a parameter grid representing the location of the parameter in the 2D model (see MATLAB documentation for further details). The initial value of damping factor $\lambda$ is based on the standard deviation of the logarithms of the apparent resistivities and lowered to half after each iteration while it is greater than 0.01 .

Following Trip et al. (1984), Sasaki (1994), and Spitzer (1998), the Jacobian matrix (J) was calculated by differentiating Eq. 2 with respect to cell conductivities, yielding:

$$
\mathbf{K} \frac{\partial \mathbf{v}}{\partial \boldsymbol{\sigma}_{\mathbf{i}}}=-\frac{\partial \mathbf{K}}{\partial \boldsymbol{\sigma}_{\mathbf{i}}} \mathbf{v} .
$$

In Eq. 8 only the partial derivatives of potentials with respect to cell conductivities are unknown. $\mathbf{K}$ and $\mathbf{v}$ matrices are already produced during the forward solution. The partial derivative of the stiffness matrix with respect to model parameters can be easily calculated by assigning 1 to relevant conductivities and 0 to all others. Because the approach of Marescot et al. (2006) is used for apparent resistivity evaluation, $\mathbf{K}$ is calculated twice. In the first 
case all conductivities are assigned as 1 and result is stored as $\mathbf{K}_{1}$. Therefore, it is sufficient to pick the values of $\mathbf{K}_{1}$ relevant to the triangles inside a parameter block to calculate its derivative with respect to a certain parameter. This is easily implemented by creating an index of triangles relating them with the parameters during the mesh creation. Equation 8 then can be handled as a linear system of equations in the form of $A x=b$ and must be solved for each model parameter. This is done as described previously for Eq. 2. In this manner, the Jacobian matrix is calculated for every iteration. An updated model is achieved by adding the calculated corrections to the model parameters. The new model is verified by comparing its response with the measured data. Data fitness is measured in the sense of RMS defined as:

$$
\mathrm{RMS}=\sqrt{\frac{\left(\mathbf{W}_{\mathrm{d}} \Delta \mathbf{d}\right)^{\mathrm{T}}\left(\mathbf{W}_{\mathrm{d}} \Delta \mathbf{d}\right)}{N},}
$$

where $N$ is the number of datum and superscript $\mathrm{T}$ denotes matrix transpose. The misfit function given in Eq. 9 is the cost function to be minimized by the inversion routine. The inversion process usually converges after 5 to 8 iterations.

For the IP data inversion I followed the third method proposed by Oldenburg and $\mathrm{Li}$ (1994). The proposed method aims to minimize the objective function given in Eq. 9 by solving a nonlinear inverse problem where the data discrepancies are now defined as

$$
\Delta d=\eta_{a m}-\eta_{a c},
$$

where $\eta_{a m}$ and $\eta_{a c}$ are the measured and calculated apparent chargeability values in $\mathrm{mV} / \mathrm{V}$, respectively. The methodology used in the inversion of apparent resistivity data is used in the same form for apparent chargeability inversion. Equation 5 is solved once more to calculate the model parameter corrections where the model vector now contains chargeability values of the model cells and the data vector is formed by the observed apparent chargeability values. The sensitivity matrix in Eq. 5 is replaced with the following one:

$$
J_{i j}^{\eta}=-\frac{\partial d_{i}}{\partial \eta_{j}}=-\sigma(1-\eta) \frac{V_{\sigma}^{i}}{\left(V_{\eta}^{i}\right)^{2}} J_{i j},
$$

where $V_{\sigma}$ and $V_{\eta}$ are the calculated potentials for conductivity and chargeability models, respectively; and $i$ and $j$ are the data and parameter indices, respectively. $J$ is the sensitivity matrix for the DC resistivity problem (Oldenburg and Li 1994). A known resistivity model is required for the evaluation of the response of a chargeability model. Therefore, the IP data 
inversion is performed sequentially after the inversion of DC resistivity data. The recovered resistivity model, the Jacobian matrix at the last successful iteration and the calculated potentials are substituted in Eqs. 4 and 10 for necessary calculations. The same misfit function is used for the verification of the recovered chargeability models.

\section{USER INTERFACE}

The user interface of ELRIS2D is designed for ease of use with all controls and options available on the same window (Fig. 2). The main window is divided into several panels. The first panel located on top left of the window is a useful and simple file explorer. As soon as the program starts, root folder (where the program is called) is scanned by a routine to find the data files with the supported file format. The data file format supported by ELRIS2D is identical to the format of well-known program RES2DInv. The program currently supports Pole-Pole, Pole-Dipole, Dipole-Dipole, Wenner, and Schlumberger arrays. The data are assumed to be measured by equally spaced electrodes. Please refer to the User Manual located in the root folder of the program for further details. Unsupported data file formats are simply

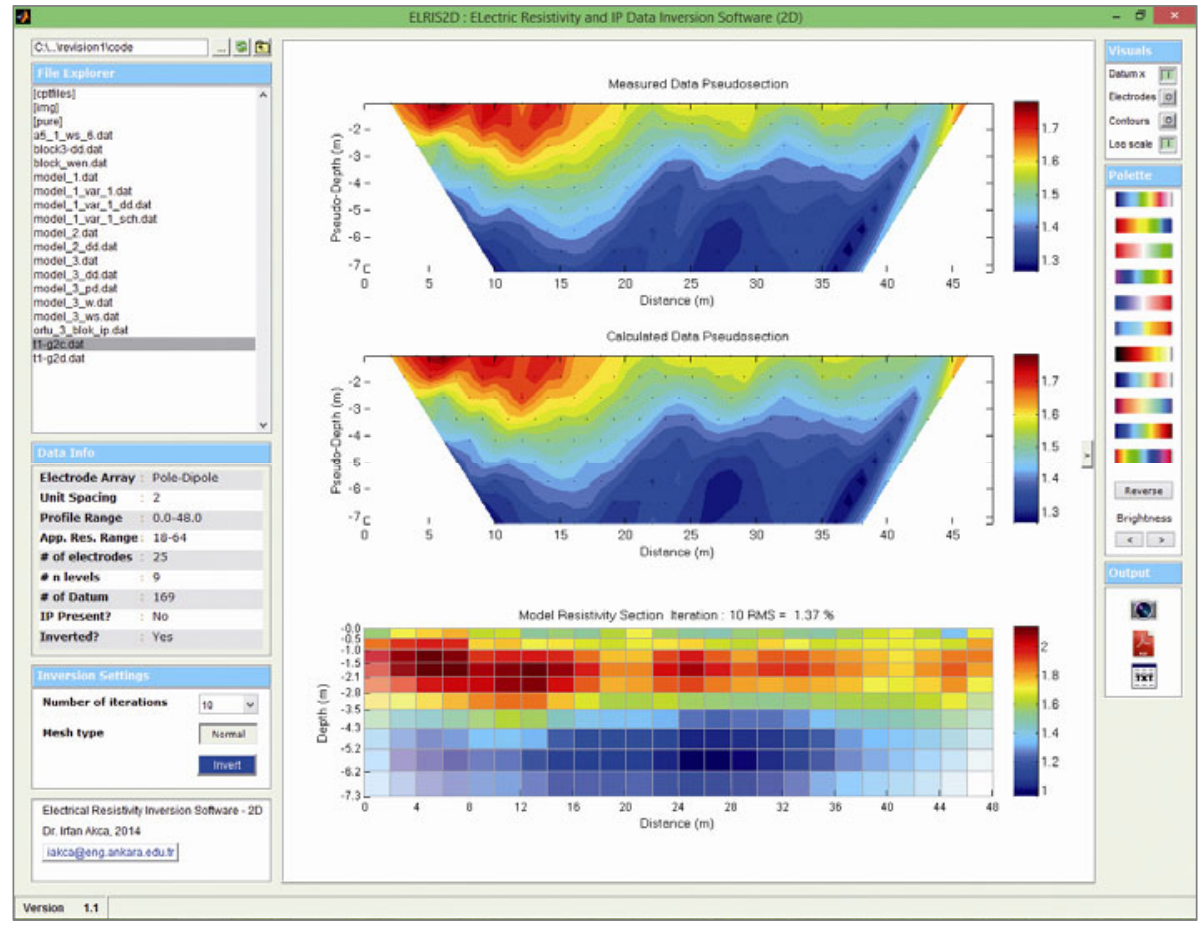

Fig. 2. The ELRIS user interface. 
not listed in the File Explorer panel. The folder names in the current directory (if any) are given at the top of the list enclosed with brackets. This list box permits some interactions:

(1) double clicking on a folder name opens and scans the selected folder for supported data files,

(2) clicking on the name of a data file directly displays the content of the file as a pseudo-section,

(3) double clicking on to a data filename displays the results of previous inversion if available.

Browse and Directory Up buttons are also located at the top of the list box for changing the working directory (Fig. 2). The Refresh button may be used to update the file list in case of copying or producing new data files. Information about a selected data file is displayed in the next panel titled as "Data Info". In this context, the user can explore the content of a file just by clicking on the filename as a pseudosection supported by basic data information.

This is more practical than using a popup menu used in the order: File $>$ Read Data $>$ Select Folder $>$ Select File $>$ Ok. If the data file has already been inverted, it is indicated in the Data Info panel and the user can recall the results by just double clicking the filename.

The Inversion Settings panel includes two options: number of iterations and the mesh type. Available mesh types are "normal" and "fine". Normal mesh corresponds to a discretization where a rectangular cell is located between each adjacent electrode. Fine mesh is constructed by dividing the space between adjacent electrodes into two rectangular cells. Inversion must be restarted after changing the mesh type.

The panels on the right hand side include most common options for displaying DCR/IP data and 2D model sections. The pseudosection plotting is done following the pseudo-depth concept defined by Edwards (1977). The $x$-location of a datum is assumed to be at the midpoint of the electrode array for a single measurement. Following this aspect, the datum coordinate is plotted on the section with a black dot. The user can toggle to display or not to display the locations of datum. In a similar way, the user has the option of displaying electrode locations. Pseudosections are plotted as color coded sections. However, the user has the option to display the contour lines as well. Resistivities of earth materials vary in a very wide range. Therefore, logarithmic color scales are favorable for a better representation of the changes in the measured data and model resistivities. An option is present in the "Visuals" panel to toggle the logarithmic and linear color scales. Any change in the available options is applied immediately to the displayed sections. 
Depending on the range of resistivity data and model resistivities, different color maps may be more suitable to obtain the best image for the interpretation. For this purpose a plenty of color schemes are made available with thumbnail previews at the palette panel. The color scales can be reversed and the darkness can be adjusted by clicking the buttons located in the palette panel.

The outcome of an inversion may be exported into other formats by three available options. A snapshot of the sections panel may be captured by snipping tool of Microsoft Windows activated by clicking a button located in output panel. Alternatively, the data and model section can be exported as a pdf file. Finally, a text file containing cell resistivities and data fitness may be generated by clicking the icons located at output panel. These options become available only when three sections are displayed in the main panel. Some other customization options are available via context menus that can be activated by right clicking on the sections. Labels, titles, length of color scales, color scale limits can be modified using several input boxes. The sections panel may be enlarged by hiding the tools located on the right hand side of the user interface by clicking the toggle button located at the right edge of the sections panel.

The current version of the code has some limitations. ELRIS supports only frequently used 5 electrode arrays listed before. Non-conventional arrays and borehole electrodes are not supported. The earth surface is assumed to be flat. The user interface of the code does not have the ability to incorporate a priori information to the inversion. However, the source code of the program is open which permits the potential users and contributors to eliminate the aforementioned limitations. Apart from the listed limitations of the present program, the following features may also be added with little effort:

$\square$ allows user to select multiple data files at a time and invert them subsequently with the user supplied settings,

a combining the inverted resistivity models of a survey site to display a pseudo three dimensional resistivity distribution.

\section{EXAMPLES}

\subsection{Test model 1}

ELRIS2D was tested with synthetic and field data sets. The first example is a synthetic data set calculated for the model given in Fig. 3d. The model consists of two embedded bodies and a laterally discontinued overburden. The resistivities of the background, overburden and embedded bodies are set as 100,250 , and $2500 \mathrm{ohm}-\mathrm{m}$, respectively. The embedded bodies simulate a cross-section of walls perpendicular to the section plane. The data were simulated for dipole-dipole electrode array with 25 equally spaced electrodes. 


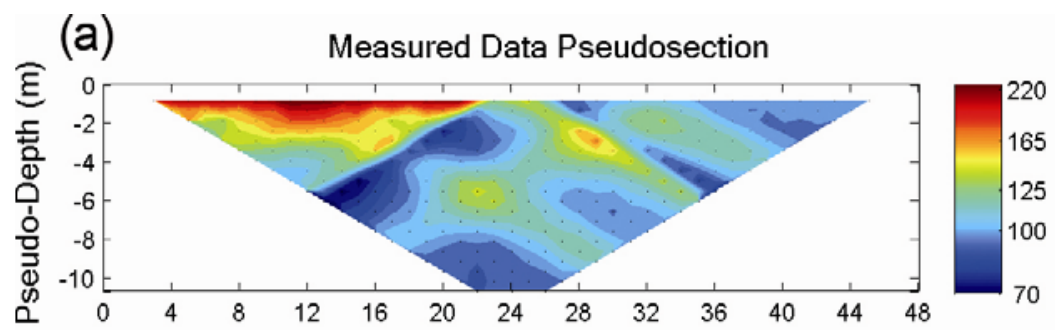

(b) Calculated Data Pseudosection

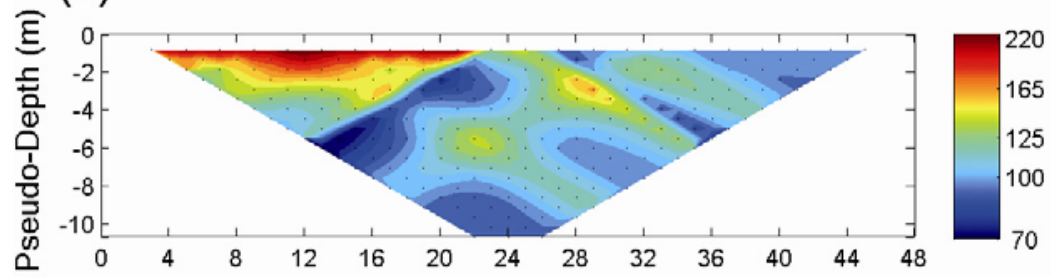

(c) Inverted Resistivity Model Iteration : 10 RMS $=1.26 \%$

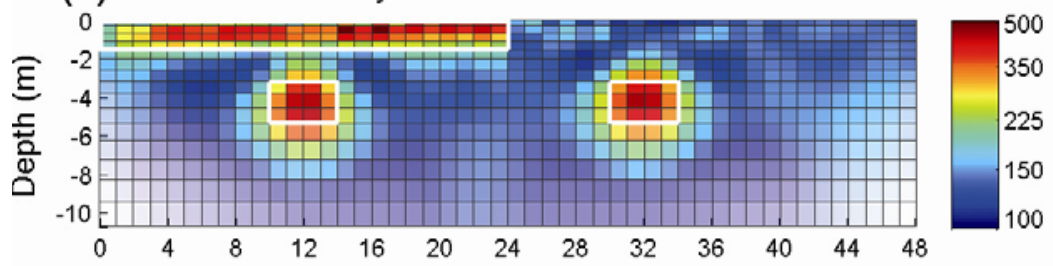

(d) Resistivity Model

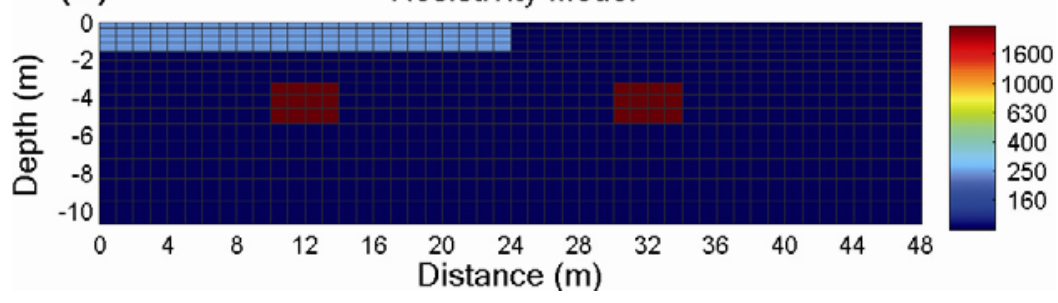

Fig. 3. Inversion results of the test model 1.

Electrode spacing was set to $2 \mathrm{~m}$. Data were contaminated with Gaussian noise of $3 \%$. Setting the response of the test model as the input data, an inversion with 10 iterations was carried out. The model mesh option was set to "fine", which means two rectangular cells are placed between each adjacent electrode. The model consists of 720 cells, each assigned an intrinsic resistivity. The expected value of RMS is 1 since the data difference is weighted. The final RMS at 10th iteration was 1.26 which is slightly above the expected error. Algorithm stops if the value of RMS tends to decrease below 1. This aims to prevent the algorithm from converging into the noise present in 
the data. The overall inversion process for 10 iterations took $25.04 \mathrm{~s}$ on a PC with Pentium Dual Core processor and 4 GB RAM. The resulting inverse model resistivity section is given in Fig. 3c. Some cells in the illustrated model are faded. Fading of the model parameters is related with the value of total sensitivity of the cell, calculated by a samovar through the columns of Jacobian matrix at first iteration. The values are normalized with the maximum sensitivity and assigned as the degree of transparency for each cell. The exact locations of the model components are marked with white rectangles in Fig. 3c. The locations and size of the bodies are well defined in the reconstructed model.

\subsection{Test model 2}

The second model was designed to test the IP data inversion capability of ELRIS2D. The test model consisted of two embedded blocks and an overburden. The background resistivity was set to $100 \mathrm{ohm}-\mathrm{m}$. Two embedded blocks simulate a conductive and a relatively resistive ore body where resistivities were set as 10 and $500 \mathrm{ohm}-\mathrm{m}$, respectively. Only the embedded blocks are chargeable $(250 \mathrm{~ms})$; therefore, the chargeability values of background and the overburden were set to 0 . The resistivity and chargeability distribution of the test model is given in Fig. $4 d$, h. The apparent resistivity and chargeability responses of the respective models are calculated assuming a multi-electrode system with 37 take-outs is used. The synthetic apparent resistivity and chargeability data are evaluated for dipole-dipole electrode configuration with $25 \mathrm{~m}$ unit electrode spacing (Fig. 4a, e). Gaussian noise of $2 \%$ was added to the simulated data.

The IP forward modeling and inversion requires a known resistivity model. Therefore, the DC resistivity response of the test model 2 is inverted first. The responses of the test model and recovered model are demonstrated in Fig. 4a, b, respectively. The recovered resistivity model is given in Fig. 4c with the exact locations of embedded bodies and the overburden marked with white rectangles. Both the conductive and resistive bodies are outlined in the inverted model section. The data misfit after 10 iterations is calculated as $2.84 \%$. The recovered model outlines the test model. The ELRIS2D automatically switches to IP inversion once the DC resistivity inversion is complete. The recovered resistivity model and the Jacobian matrix at the last successful iteration are passed to the IP inversion routine for necessary calculations. IP data inversion is straightforward because the previously created model mesh is used and the former routines are called with small modifications. ELRIS2D enables a radio button group whenever a data file containing apparent chargeability data is selected. User can switch between resistivity and chargeability sections by selecting appropriate option 

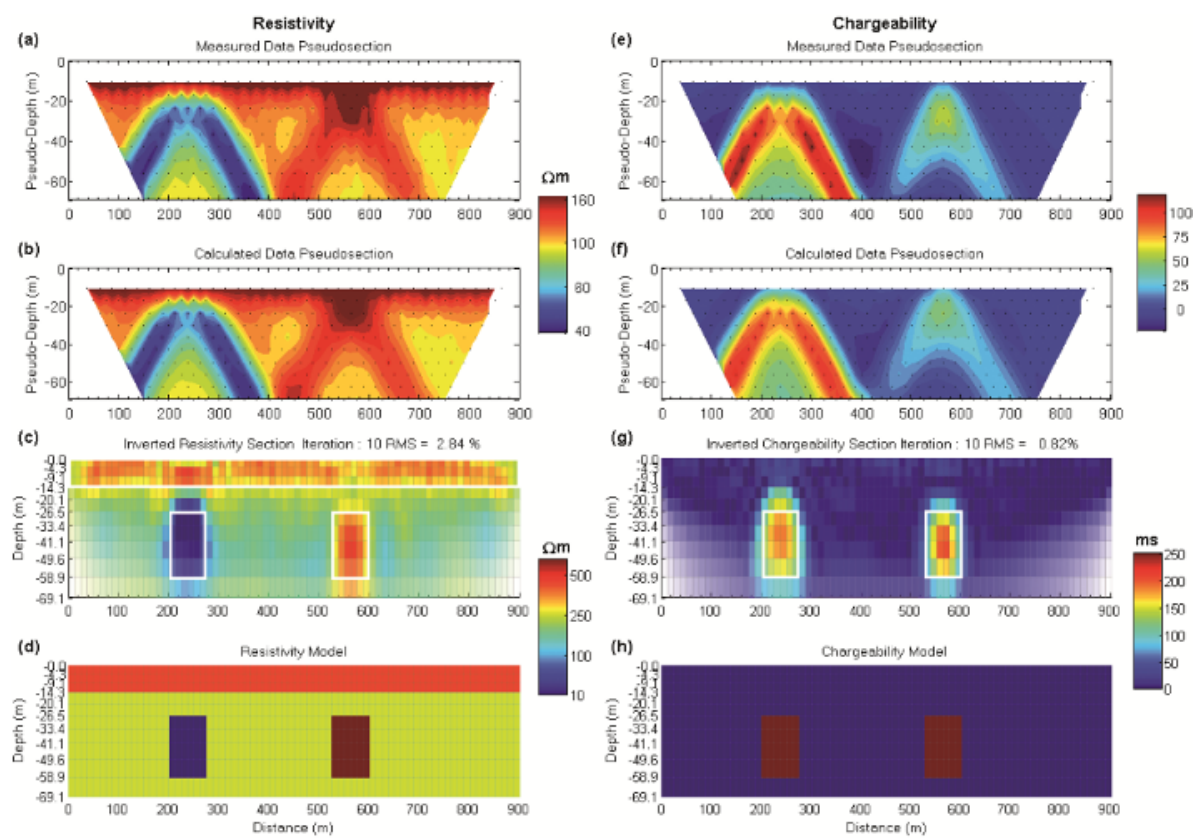

Fig. 4. The inversion results of test model 2. The left panel is for resistivity data and models, while chargeability data and models are shown on the right.

on the radio button group placed at the bottom of the sections panel. The inversion results of the IP data are demonstrated in the axes placed on the right panel of Fig. 4. The model and data space for IP inversion is linear. The recovered chargeability model is demonstrated in Fig. $4 \mathrm{~g}$ with the exact location of embedded blocks marked with white rectangles. Remember that only the embedded blocks were chargeable and the background chargeability was set to zero. By visual comparison of the actual and recovered chargeability models it may be stated that the algorithm has the ability of outlining the position and chargeability of the anomalous bodies. This conclusion is supported with the fitness between the measured and calculated data sets. The apparent chargeability values are noise free and the data space is linear. Therefore, the data fitness is calculated as $0.82 \%$ after 10 iterations.

\subsection{Field data test}

The algorithm was also tested with field data sets. Field data were measured at the ancient city of Pisidian Antioch (Öztürk Akca 2011) located nearby the town of Yalvaç in southwest Turkey (Fig. 5a). The significant part of the city is still not excavated (Fig. 5b). Therefore, ERT measurements were carried out for the purpose of archaeological prospection. Measurements were 


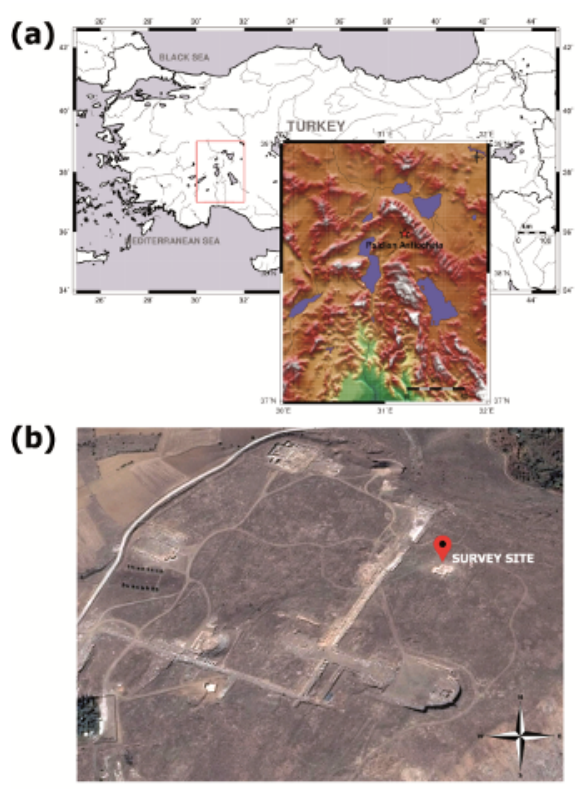

(c)
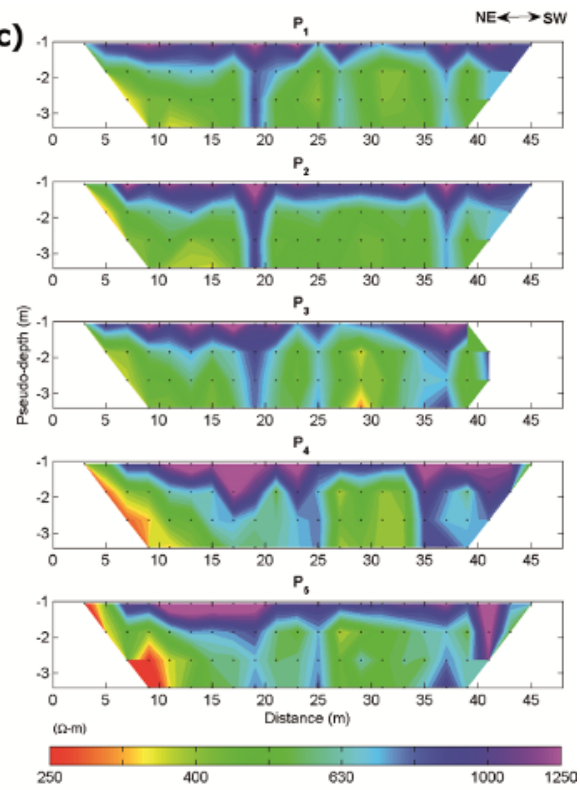

Fig. 5: (a) Location of survey area, (b) general view of the survey site, and (c) field data measured at Aedilis Hill.

taken by a multi-electrode system with 25 electrodes. The field data set was measured on the hill called "Aedilis" overlooking the city (Fig. 5b). Archaeologists expected the existence of a kind of temple due to the location of the hill. Therefore, a preliminary survey was carried out at the mentioned location. A Wenner-Schlumberger array with $2 \mathrm{~m}$ electrode spacing was used to measure the data on five parallel profiles $2 \mathrm{~m}$ apart. Profiles are numbered $\mathrm{P} 1$ to P5, starting from the one located at NW. The apparent resistivity pseudosections of the measured lines are demonstrated in Fig. 5c.

The data were inverted using a fine mesh consisting of 288 parameters. The number of iterations was set to 10. All data were inverted using the same settings. Data misfits varied between 5 to $11 \%$. Inverted model resistivity sections of all profiles are illustrated in Fig. 6a with same color range and a common logarithmic scale. Two main anomalous bodies can be distinguished from the background. The first one is located at a distance of around $20 \mathrm{~m}$ from the beginning of the lines. The strength of the anomaly reaches to $2000 \mathrm{ohm}-\mathrm{m}$ where the background resistivity is assumed to be around $500 \mathrm{ohm}-\mathrm{m}$. The second anomaly is located between 36 to $42 \mathrm{~m}$ of the lines and may be interpreted as two close embedded bodies. The overall inspection of the resistivity sections proves the existence of the remains of an ancient structure. The study was a preliminary examination of the survey area; 

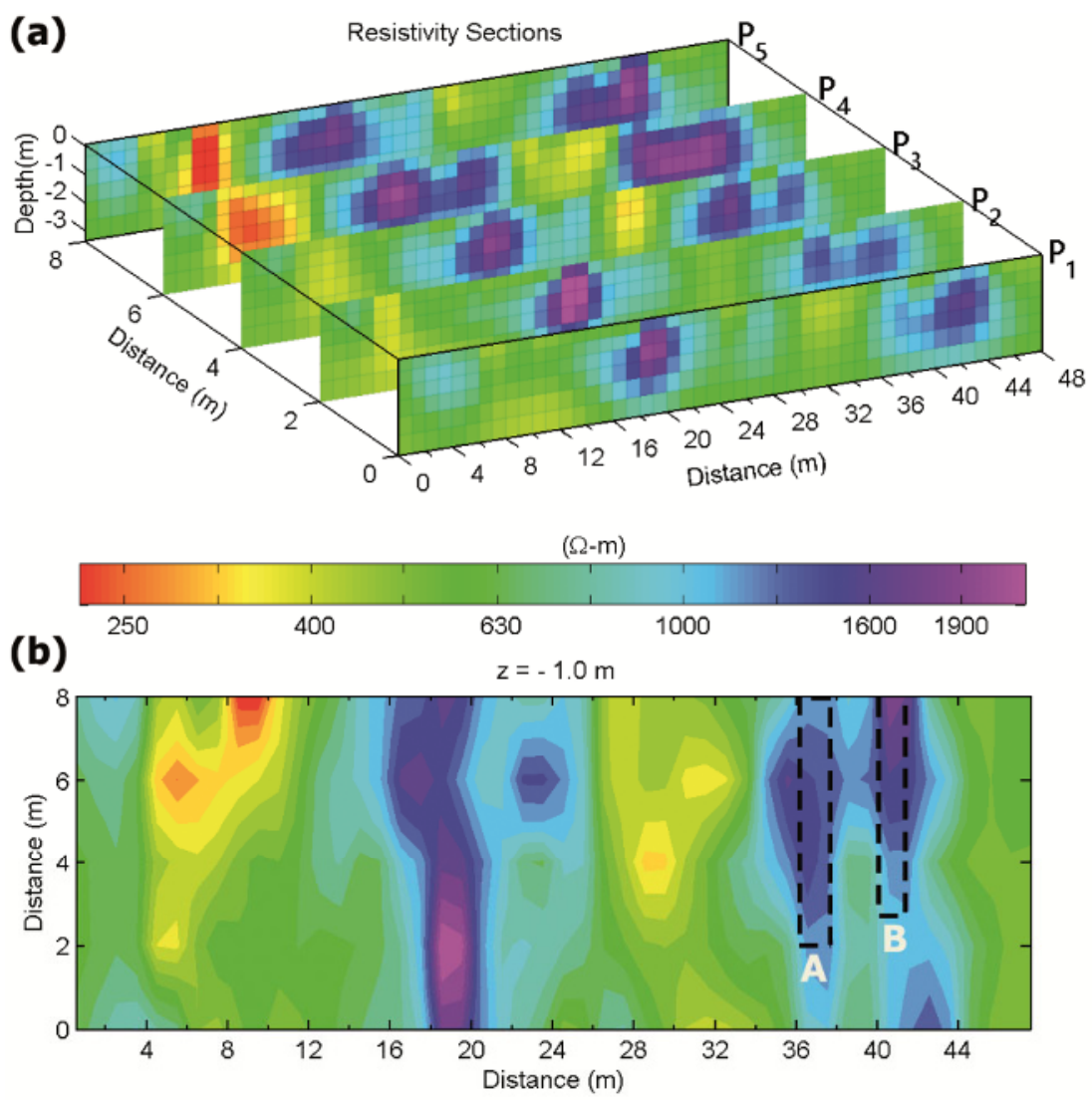

(c)

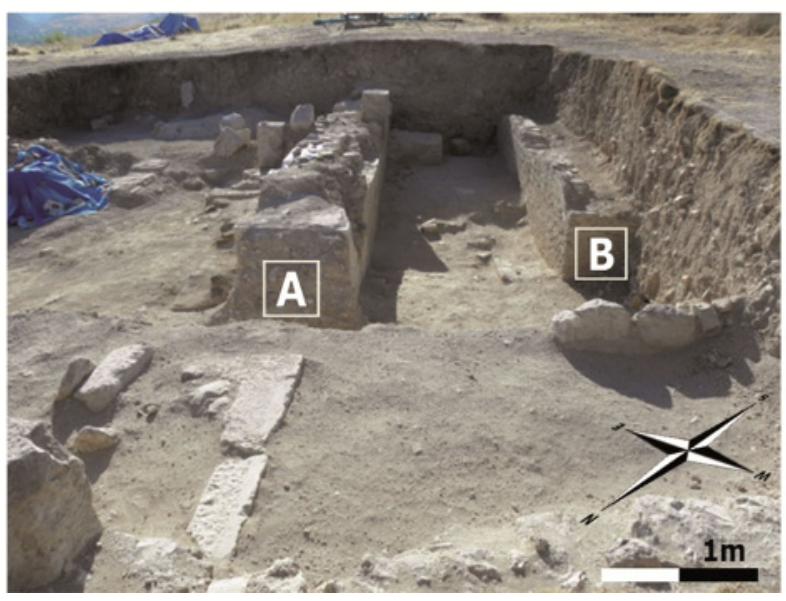

Fig. 6. Inversion results of field data: (a) model resistivity sections, (b) resistivity map of $z=-1.0 \mathrm{~m}$ depth, and (c) excavation result at survey site. 
therefore, the electrode and profile spacing were set to $2 \mathrm{~m}$ which is quite large for an archaeogeophysical survey. The survey site was excavated during the studies in 2011, which resulted in the exploration of the sixth church of the ancient city (Özhanl, private communication). Figure $6 \mathrm{c}$ shows a picture of the excavation area. The anomaly located between 36 to $42 \mathrm{~m}$ of the lines corresponded to two walls made of limestone $2 \mathrm{~m}$ apart. The depth of the top of walls was $1.25 \mathrm{~m}$ from the surface. The dimension and locations of the walls are marked on the resistivity map of $1.0 \mathrm{~m}$ depth given in Fig. $6 \mathrm{~b}$. The other anomaly, clearly seen around $18-20 \mathrm{~m}$, possibly arose from another structure, such as a wall. However, this interpretation has not verified because that part of the survey area is still unexcavated.

\section{CONCLUSIONS}

Two-dimensional inversion of resistivity and IP data is a well-established research area in geophysical community. Several commercial and noncommercial inversion codes are produced with good coverage of available inversion and visualization options. Emerging capabilities of measuring devices produce large amount of data in shorter times. Therefore, practical inversion programs are needed for the fast and efficient interpretation of acquired data sets. A new MATLAB program named ELRIS2D was developed for the two-dimensional inversion of DC resistivity and IP data to meet these demands. Two main new features of the program are the approach used to construct the model mesh and the practical user interface. Both features help to reduce the computation requirements and the time spent by the interpreter to process multiple data sets. The interactive file list box permits switching between data files in the current folder by just a mouse click or using arrow keys. Content of selected data files and previous inversion results (if available) are displayed immediately by appropriate mouse clicks. The inversion algorithm is based on the smoothness constrained least squares scheme. Based on the availability, program automatically inverts the apparent chargeability data whenever a resistivity data inversion is completed. A non-linear scheme is used to invert IP data. Switching between resistivity/ chargeability sections are as easy as clicking a radio button place on the user interface. The program provided satisfactory processing times $(\sim 1.5 \mathrm{~seconds} /$ iteration for a moderate model although the Jacobian matrix is recalculated at each iteration).

ELRIS2D is an open source program which is expected to be improved by potential users and developers. Support for including topography in the inversion, simultaneous visualization of multiple sections, more visual and inversion options may be added for the next releases. The current version of the program is verified by two test models and a set of field data. 


\subsection{Availability and requirements}

The source code and supplementary files of the program are available at https://goo.gl/PZ2tMC as a compressed folder. There is no specific setup procedure. It will be enough to unzip the contents of the compressed folder to a specific location. The program may be started by calling the main function elris.m from the MATLAB command line. The source code was developed on a PC operated by Windows 7 (64 bit) equipped with 4 GB RAM and a Pentium dual core processor. The program was tested on the MATLAB versions R2010a and R2013b resulting in a full functionality. The MATLAB version R2010a and newer must be installed on the user's computer. There is no strict hardware requirements, however it is suggested to have at least 3 GB of RAM installed. The data format and usage details are given in the user manual included in the main folder.

Acknowledgments. The author would like to thank Dr. Cemile Öztürk Akca for providing the field data measured during her Ph.D. study. The measurement system was belonging to Ankara University Geophysical Engineering Department. The author would like to thank head of department for permitting to use the equipment. Some functions (othercolor.m, cptcmap.m, imageviewer.m) used in the program are downloaded from the MATLAB File Exchange server. I would like to thank the authors of the functions for sharing their work.

\section{References}

Akca, I., and A.T. Başokur (2010), Extraction of structure-based geoelectric models by hybrid genetic algorithms, Geophysics 75, 1, F15-F22, DOI: 10.1190/ 1.3273851 .

Başokur, A.T., and I. Akca (2011), Object-based model verification by a genetic algorithm approach: Application in archeological targets, J. Appl. Geophys. 74, 4, 167-174, DOI: 10.1016/j.jappgeo.2011.05.004.

Bertin, J., and J. Loeb (1976), Experimental and Theoretical Aspects of Induced Polarization, Vols. 1 and 2, Gebrüder Borntraeger, Berlin.

Coggon, J.H. (1971), Electromagnetic and electrical modeling by the finite element method, Geophysics 36, 1, 132-155, DOI: 10.1190/1.1440151.

DC2DInvRes (2014), DC2DInvRes - Tutorial, http://www. resistivity.net.

Dey, A., and H.F. Morrison (1979), Resistivity modelling for arbitrarily shaped twodimensional structures, Geophys. Prospect. 27, 1, 106-136, DOI: 10.1111/ j.1365-2478.1979.tb00961.x.

EarthImager (2009) EarthImager 2D. Resistivity and IP inversion software. Instruction manual, Advanced Geosciences, Inc., Austin, USA, 139 pp. 
Edwards, L.S. (1977), A modified pseudosection for resistivity and IP, Geophysics 42, 5, 1020-1036, DOI: 10.1190/1.1440762.

Farquharson, C.G., and D.W. Oldenburg (1998), Non-linear inversion using general measures of data misfit and model structure, Geophys. J. Int. 134, 1, 213227, DOI: 10.1046/j.1365-246x.1998.00555.x.

Fink, J.B., E.O. McAlister, B.K. Sternberg, W.G. Wieduwilt, and S.H. Ward (eds.) (1990), Induced Polarization: Applications and case histories, Investigations in Geophysics, No. 4, Society of Exploration Geophysicists, Tulsa, DOI: $10.1190 / 1.9781560802594$.

Günther, T. (2004), Inversion methods and resolution analysis for the 2D/3D reconstruction of resistivity structures from DC measurements, Ph.D. Thesis, Technische Universitaet Bergakademie, Freiberg, Germany.

Günther, T., and C. Rücker (2015), Boundless Electrical Resistivity Tomography BERT 2 - the user tutorial, http://resistivity.net/download/bert-tutorial.pdf.

Karaoulis, M., A. Revil, P. Tsourlos, D.D. Werkema, and B.J. Minsley (2013), IP4DI: A software for time-lapse 2D/3D DC-resistivity and induced polarization tomography, Comput. Geosci. 54, 164-170, DOI: 10.1016/j.cageo. 2013.01.008.

Loke, M.H. (2014), Tutorial: 2-D and 3-D electrical imaging surveys, http://www. geotomosoft.com/coursenotes.zip.

Loke, M.H., and R.D. Barker (1996), Rapid least-squares inversion of apparent resistivity pseudosections by a quasi-Newton method, Geophys. Prospect. 44, 1, 131-152, DOI: 10.1111/j.1365-2478.1996.tb00142.x.

Loke, M.H., J.E. Chambers, D.F. Rucker, O. Kuras, and P.B. Wilkinson (2013), Recent developments in the direct-current geoelectrical imaging method, J. Appl. Geophys. 95, 135-156, DOI: 10.1016/j.jappgeo.2013.02.017.

Marescot, L., S. Rigobert, S.P. Lopes, R. Lagabrielle, and D. Chapellier (2006), A general approach for DC apparent resistivity evaluation on arbitrarily shaped 3D structures, J. Appl. Geophys. 60, 1, 55-67, DOI: 10.1016/ j.jappgeo.2005.12.003.

Mufti, I.R. (1976), Finite-difference resistivity modeling for arbitrarily shaped twodimensional structures, Geophysics 41, 1, 62-78, DOI: 10.1190/1.1440608.

Oldenburg, D.W., and Y. Li (1994), Inversion of induced polarization data, Geophysics 59, 9, 1327-1341, DOI: 10.1190/1.1443692.

Öztürk Akca, C. (2011), Geoarcheological and new archaeogeopyhsical methods applied at ancient settlements: Psidia Antiocheia example, Ph.D. Thesis, Süleyman Demirel University, Isparta, Turkey.

Pelton, W.H., L. Rijo, and C.M. Swift (1978), Inversion of two-dimensional resistivity and induced-polarization data, Geophysics 43, 4, 788-803, DOI: 10.1190/1.1440854. 
Pidlisecky, A., and R. Knight (2008), FW2_5D: A MATLAB 2.5-D electrical resistivity modeling code, Comput. Geosci. 34, 12, 1645-1654, DOI: 10.1016/ j.cageo.2008.04.001.

Pidlisecky, A., E. Haber, and R. Knight (2007), RESINVM3D: A 3D resistivity inversion package, Geophysics 72, 2, H1-H10, DOI: 10.1190/1.2402499.

Res2DInv (2014), Res2DInv user manual. Version 4.03, Geotomo Software, Penang, Malaysia.

Rijo, L. (1977), Modeling of electric and electromagnetic data, Ph.D. Thesis, University of Utah, Salt Lake City, USA.

Rücker, C. (2011), Advanced electrical resistivity modelling and inversion using unstructured discretization, Ph.D. Thesis, Leipzig University, Germany.

Sasaki, Y. (1994), 3-D resistivity inversion using the finite-element method, Geophysics 59, 12, 1839-1848, DOI: 10.1190/1.1443571.

Seigel, H.O. (1959), Mathematical formulation and type curves for induced polarization, Geophysics 24, 3, 547-565, DOI: 10.1190/1.1438625.

Shewchuk, R.J. (1997), Delaunay refinement mesh generation, Ph.D. Thesis, Carnegie Mellon University, Pittsburgh, USA.

Si, H. (2008), Three dimensional boundary conforming Delaunay mesh generation, $\mathrm{Ph} . \mathrm{D}$. Thesis, Inst. für Mathematik, Technische Universitat Berlin, Germany.

Spitzer, K. (1998), The three-dimensional DC sensitivity for surface and subsurface sources, Geophys. J. Int. 134, 3, 736-746, DOI: 10.1046/j.1365-246x.1998. 00592.x.

Sumner, J.S. (1976), Principles of Induced Polarization for Geophysical Exploration, Developments in Economic Geology, Vol. 5, Elsevier Science Publ. Co., Amsterdam.

Tripp, A.C., G.W. Hohmann and C.M. Swift (1984), Two-dimensional resistivity inversion, Geophysics 49, 10, 1708-1717, DOI: 10.1190/1.1441578.

Tsourlos, P.I., J.E. Szymanski, and G.N. Tsokas (1998), A smoothness constrained algorithm for the fast 2-D inversion of DC resistivity and induced polarization data, J. Balkan Geophys. Soc. 1, 1, 3-13.

Ward, S.H. (ed.) (1990), Geotechnical and Environmental Geophysics, Vols. 1-3, Investigations in Geophysics, No. 5, Soc. of Explor. Geophysicists, Tulsa.

Wolke, R., and H. Schwetlick (1988), Iteratively reweighted least squares: algorithms, convergence analysis, and numerical comparisons, SIAM J. Sci. Stat. Comput. 9, 5, 907-921, DOI: 10.1137/0909062.

Zhou, J., A. Revil, M. Karaoulis, D. Hale, J. Doetsch, and S. Cuttler (2014), Imageguided inversion of electrical resistivity data, Geophys. J. Int. 197, 1, 292309, DOI: $10.1093 / \mathrm{gji} / \mathrm{ggu} 001$. 